\title{
Skew Littlewood-Richardson rules from Hopf algebras
}

\author{
Thomas Lam1"; Aaron Lauve ${ }^{2}$, and Frank Sottile" \\ ${ }^{1}$ Department of Mathematics \\ University of Michigan \\ Ann Arbor, MI 48109 \\ ${ }^{2}$ Department of Mathematics \\ Texas A\&M University \\ College Station, TX 77843
}

\begin{abstract}
We use Hopf algebras to prove a version of the Littlewood-Richardson rule for skew Schur functions, which implies a conjecture of Assaf and McNamara. We also establish skew Littlewood-Richardson rules for Schur $P$ - and $Q$-functions and noncommutative ribbon Schur functions, as well as skew Pieri rules for $k$-Schur functions, dual $k$-Schur functions, and for the homology of the affine Grassmannian of the symplectic group.

Résumé. Nous utilisons des algèbres de Hopf pour prouver une version de la règle de Littlewood-Richardson pour les fonctions de Schur gauches, qui implique une conjecture d'Assaf et McNamara. Nous établissons également des règles de Littlewood-Richardson gauches pour les $P$ - et $Q$-fonctions de Schur et les fonctions de Schur rubbans non commutatives, ainsi que des règles de Pieri gauches pour les $k$-fonctions de Schur, les $k$-fonctions de Schur duales, et pour l'homologie de la Grassmannienne affine du groupe symplectique.
\end{abstract}

Keywords: symmetric functions, Littlewood-Richardson rule, Pieri rule, Hopf algebras, antipode

Assaf and McNamara [AM] recently used combinatorics to give an elegant and surprising formula for the product of a skew Schur function and a complete homogeneous symmetric function. Their paper included a conjectural skew version of the Littlewood-Richardson rule, and also an appendix by one of us (Lam) with a simple algebraic proof of their formula. We show how these formulas and much more are special cases of a simple formula that holds for any pair of dual Hopf algebras. We first establish this Hopf-algebraic formula, and then apply it to obtain formulas in some well-known Hopf algebras in combinatorics.

\section{A Hopf algebraic formula}

We assume basic familiarity with Hopf algebras, as found in the opening chapters of the book [Mon93]. Let $H, H^{*}$ be a pair of dual Hopf algebras over a field $\mathbb{k}$. This means that there is a nondegenerate pairing

\footnotetext{
${ }^{\dagger}$ Supported by a Sloan Fellowship, and by NSF grants DMS-0901111 and DMS-0652641.

$\ddagger$ Supported by the NSF grant DMS-0701050. 
$\langle\cdot, \cdot\rangle: H \otimes H^{*} \rightarrow \mathbb{k}$ for which the structure of $H^{*}$ is dual to that of $H$ and vice-versa. For example, $H$ could be finite-dimensional and $H^{*}$ its linear dual, or $H$ could be graded with each component finitedimensional and $H^{*}$ its graded dual. These algebras naturally act on each other [Mon93, 1.6.5]: suppose that $h \in H$ and $a \in H^{*}$ and set

$$
h \rightarrow a:=\sum\left\langle h, a_{2}\right\rangle a_{1} \quad \text { and } \quad a \rightarrow h:=\sum\left\langle h_{2}, a\right\rangle h_{1} .
$$

(We use Sweedler notation for the coproduct, $\Delta h=\sum h_{1} \otimes h_{2}$.) These left actions are the adjoints of right multiplication: for $g, h \in H$ and $a, b \in H^{*}$,

$$
\langle g, h \rightarrow a\rangle=\langle g \cdot h, a\rangle \quad \text { and } \quad\langle a \rightarrow h, b\rangle=\langle h, b \cdot a\rangle .
$$

This shows that $H^{*}$ is a left $H$-module under the action in (1). In fact, $H^{*}$ is a left $H$-module algebra, meaning that for $a, b \in H^{*}$ and $h \in H$,

$$
h \rightarrow(a \cdot b)=\sum\left(h_{1} \rightarrow a\right) \cdot\left(h_{2} \rightarrow b\right) .
$$

Recall that the counit $\varepsilon: H \rightarrow \mathbb{k}$ and antipode $S: H \rightarrow H$ satisfy $\sum h_{1} \cdot \varepsilon\left(h_{2}\right)=h$ and $\sum h_{1} \cdot S\left(h_{2}\right)=$ $\varepsilon(h) \cdot 1_{H}$ for all $h \in H$.

Lemma 1 For $g, h \in H$ and $a \in H^{*}$, we have

$$
(a \rightarrow g) \cdot h=\sum\left(S\left(h_{2}\right) \rightarrow a\right) \rightarrow\left(g \cdot h_{1}\right) .
$$

Proof: Let $b \in H^{*}$. We prove first the formula

$$
(h \rightarrow b) \cdot a=\sum h_{1} \rightarrow\left(b \cdot\left(S\left(h_{2}\right) \rightarrow a\right)\right) .
$$

(This is essentially $(*)$ in the proof of Lemma 2.1.4 in [Mon93].) Expanding the sum using [2] and coassociativity, $(\Delta \otimes 1) \circ \Delta(h)=(1 \otimes \Delta) \circ \Delta(h)=\sum h_{1} \otimes h_{2} \otimes h_{3}$, gives

$$
\begin{aligned}
\sum h_{1} \rightarrow\left(b \cdot\left(S\left(h_{2}\right) \rightarrow a\right)\right) & =\sum\left(h_{1} \rightarrow b\right) \cdot\left(h_{2} \rightarrow\left(S\left(h_{3}\right) \rightarrow a\right)\right) \\
& =\sum\left(h_{1} \rightarrow b\right) \cdot\left(\left(h_{2} \cdot S\left(h_{3}\right)\right) \rightarrow a\right) \\
& =(h \rightarrow b) \cdot a .
\end{aligned}
$$

Here, (5) follows as $H^{*}$ is an $H$-module and (6) from the antipode and counit conditions.

Note that $\langle(a \rightarrow g) \cdot h, b\rangle=\langle a \rightarrow g, h \rightarrow b\rangle=\langle g,(h \rightarrow b) \cdot a\rangle$. Using (4) this becomes

$$
\begin{aligned}
\left\langle g, \sum h_{1} \rightarrow\left(b \cdot\left(S\left(h_{2}\right) \rightarrow a\right)\right)\right\rangle & =\sum\left\langle g \cdot h_{1}, b \cdot\left(S\left(h_{2}\right) \rightarrow a\right)\right\rangle \\
& =\left\langle\sum\left(S\left(h_{2}\right) \rightarrow a\right) \rightarrow\left(g \cdot h_{1}\right), b\right\rangle,
\end{aligned}
$$

which proves the lemma, as this holds for all $b \in H^{*}$.

Remark 2 This proof is identical to the argument in the appendix to [AM], where $h$ was a complete homogeneous symmetric function in the Hopf algebra $H$ of symmetric functions. 


\section{Application to distinguished bases}

We apply Lemma 1 to produce skew Littlewood-Richardson rules for several Hopf algebras in algebraic combinatorics. We isolate the common features of those arguments.

In the notation of Section 11 , let $\left\{L_{\lambda}\right\} \subset H$ and $\left\{R_{\lambda}\right\} \subset H^{*}$ be dual bases indexed by some set $\mathcal{P}$, so $\left\langle L_{\lambda}, R_{\mu}\right\rangle=\delta_{\lambda, \mu}$ for $\lambda, \mu \in \mathcal{P}$. Define structure constants for $H$ and $H^{*}$ via

$$
\begin{array}{rlrl}
L_{\lambda} \cdot L_{\mu} & =\sum_{\nu} b_{\lambda, \mu}^{\nu} L_{\nu} & \Delta\left(L_{\nu}\right)=\sum_{\lambda, \mu} c_{\lambda, \mu}^{\nu} L_{\lambda} \otimes L_{\mu}=\sum_{\mu} L_{\nu / \mu} \otimes L_{\mu} \\
R_{\lambda} \cdot R_{\mu}=\sum_{\nu} c_{\lambda, \mu}^{\nu} R_{\nu} & \Delta\left(R_{\nu}\right)=\sum_{\lambda, \mu} b_{\lambda, \mu}^{\nu} R_{\lambda} \otimes R_{\mu}=\sum_{\mu} R_{\nu / \mu} \otimes R_{\mu} .
\end{array}
$$

The skew elements $L_{\nu / \mu}$ and $R_{\nu / \mu}$ defined above co-multiply according to

$$
\Delta\left(L_{\tau / \sigma}\right)=\sum_{\pi, \rho} c_{\pi, \rho, \sigma}^{\tau} L_{\pi} \otimes L_{\rho} \quad \Delta\left(R_{\tau / \sigma}\right)=\sum_{\pi, \rho} b_{\pi, \rho, \sigma}^{\tau} R_{\pi} \otimes R_{\rho} .
$$

(Note that the structure of $H^{*}$ can be recovered from the structure of $H$. Thus, we may suppress the analogs of (8) and the second formula in $(9)$ in the coming sections.)

Finally, suppose that the antipode acts on $H$ in the $L$-basis according to the formula

$$
S\left(L_{\rho}\right)=(-1)^{\mathrm{e}(\rho)} L_{\rho^{\top}}
$$

for some functions e: $\mathcal{P} \rightarrow \mathbb{N}$ and $(\cdot)^{\top}: \mathcal{P} \rightarrow \mathcal{P}$. Then Lemma 1 takes the following form.

Theorem 3 (Algebraic Littlewood-Richardson formula) For any $\lambda, \mu, \sigma, \tau \in \mathcal{P}$, we have

$$
L_{\mu / \lambda} \cdot L_{\tau / \sigma}=\sum_{\pi, \rho, \lambda^{-}, \mu^{+}}(-1)^{\mathrm{e}(\rho)} c_{\pi, \rho, \sigma}^{\tau} b_{\lambda^{-}, \rho^{\mathrm{T}}}^{\lambda} b_{\mu, \pi}^{\mu^{+}} L_{\mu^{+} / \lambda^{-}} .
$$

Swapping $L \leftrightarrow R$ and $b \leftrightarrow c$ in (11) yields the analog for the skew elements $R_{\mu / \lambda}$ in $H^{*}$.

Proof: The actions in (1) together with the second formulas for the coproducts in (7) and (8) show that $R_{\lambda} \rightarrow L_{\mu}=L_{\mu / \lambda}$ and $L_{\lambda} \rightarrow R_{\mu}=R_{\mu / \lambda}$. Now use (3) and (7)-(10) to obtain

$$
\begin{aligned}
L_{\mu / \lambda} \cdot L_{\tau / \sigma}=\left(R_{\lambda} \rightarrow L_{\mu}\right) \cdot L_{\tau / \sigma} & =\sum_{\pi, \rho}(-1)^{\mathrm{e}(\rho)} c_{\pi, \rho, \sigma}^{\tau}\left(\left(L_{\rho^{\top}} \rightarrow R_{\lambda}\right) \rightarrow\left(L_{\mu} \cdot L_{\pi}\right)\right) \\
& =\sum_{\pi, \rho, \mu^{+}}(-1)^{\mathrm{e}(\rho)} c_{\pi, \rho, \sigma}^{\tau} b_{\mu, \pi}^{\mu^{+}}\left(R_{\lambda / \rho^{\top}} \rightarrow L_{\mu^{+}}\right) \\
& =\sum_{\pi, \rho, \lambda^{-}, \mu^{+}}(-1)^{\mathrm{e}(\rho)} c_{\pi, \rho, \sigma}^{\tau} b_{\lambda^{-}, \rho^{\top}}^{\lambda} b_{\mu, \pi}^{\mu^{+}}\left(R_{\lambda^{-}} \rightarrow L_{\mu^{+}}\right) .
\end{aligned}
$$

This equals the right hand side of (11), since $R_{\lambda^{-}} \rightarrow L_{\mu^{+}}=L_{\mu^{+} / \lambda^{-}}$.

Remark 4 The condition (10) is highly restrictive. It implies that the antipode $S$, as a linear map, is conjugate to a signed permutation matrix. Nevertheless, it holds for the Hopf algebras we consider. More generally, it holds if either $H$ or $H^{*}$ is commutative, for then $S$ is an involution [Mon93, Cor. 1.5.12]. 


\section{Skew Littlewood-Richardson rule for Schur functions}

The commutative Hopf algebra $\Lambda$ of symmetric functions is graded and self-dual under the Hall inner product $\langle\cdot, \cdot\rangle: \Lambda \otimes \Lambda \rightarrow \mathbb{Q}$. A systematic study of $\Lambda$ from a Hopf algebra perspective appears in [Zel81]. We follow the definitions and notation in Chapter I of [Mac95]. The Schur basis of $\Lambda$ (indexed by partitions) is self-dual, so (7) and (9) become

$$
\begin{gathered}
s_{\lambda} \cdot s_{\mu}=\sum_{\nu} c_{\lambda, \mu}^{\nu} s_{\nu}\left(s_{\nu}\right)=\sum_{\lambda, \mu} c_{\lambda, \mu}^{\nu} s_{\lambda} \otimes s_{\mu}=\sum_{\mu} s_{\nu / \mu} \otimes s_{\mu} \\
\Delta\left(s_{\tau / \sigma}\right)=\sum_{\pi, \rho} c_{\pi, \rho, \sigma}^{\tau} s_{\pi} \otimes s_{\rho},
\end{gathered}
$$

where the $c_{\lambda, \mu}^{\nu}$ are the Littlewood-Richardson coefficients and the $s_{\nu / \mu}$ are the skew Schur functions [Mac95, I.5]. Combinatorial expressions for the $c_{\lambda, \mu}^{\nu}$ and inner products $\left\langle s_{\mu / \lambda}, s_{\tau / \sigma}\right\rangle$ are derived using the Hopf algebraic structure of $\Lambda$ in [Zel81]. The coefficients $c_{\pi, \rho, \sigma}^{\tau}$ occur in the triple product $s_{\pi} \cdot s_{\rho} \cdot s_{\sigma}$,

$$
c_{\pi, \rho, \sigma}^{\tau}=\left\langle s_{\pi} \cdot s_{\rho} \cdot s_{\sigma}, s_{\tau}\right\rangle=\left\langle s_{\pi} \cdot s_{\rho}, s_{\tau / \sigma}\right\rangle=\left\langle s_{\pi} \otimes s_{\rho}, \Delta\left(s_{\tau / \sigma}\right)\right\rangle .
$$

Write $\rho^{\prime}$ for the conjugate (matrix-transpose) of $\rho$. Then the action of the antipode is

$$
S\left(s_{\rho}\right)=(-1)^{|\rho|} s_{\rho^{\prime}},
$$

which is just a twisted form of the fundamental involution $\omega$ that sends $s_{\rho}$ to $s_{\rho^{\prime}}$. Indeed, the formula $\sum_{i+j=n}(-1)^{i} e_{i} h_{j}=\delta_{0, n}$ shows that (14) holds on the generators $\left\{h_{n} \mid n \geq 1\right\}$ of $\Lambda$. The validity of (14) follows as both $S$ and $\omega$ are algebra maps.

Since $c_{\lambda^{-}, \rho^{\prime}}^{\lambda}=0$ unless $|\rho|=\left|\lambda / \lambda^{-}\right|$, we may write (11) as

$$
s_{\mu / \lambda} \cdot s_{\tau / \sigma}=\sum_{\pi, \rho, \lambda^{-}, \mu^{+}}(-1)^{\left|\lambda / \lambda^{-}\right|} c_{\pi, \rho, \sigma}^{\tau} c_{\lambda^{-}, \rho^{\prime}}^{\lambda} c_{\mu, \pi}^{\mu^{+}} s_{\mu^{+} / \lambda^{-}} .
$$

We next formulate a combinatorial version of (15). Given partitions $\rho$ and $\sigma$, form the skew shape $\rho * \sigma$ by placing $\rho$ southwest of $\sigma$. Thus,

$$
\text { if } \rho=\boxplus \text { and } \sigma=\Psi^{\text {then }} \rho * \sigma=\boxplus^{\square} \text {. }
$$

Similarly, if $R$ is a tableau of shape $\rho$ and $S$ a tableau of shape $\sigma$, then $R * S$ is the skew tableau of shape $\rho * \sigma$ obtained by placing $R$ southwest of $S$. Fix a tableau $T$ of shape $\tau$. The Littlewood-Richardson coefficient $c_{\rho, \sigma}^{\tau}$ is the number of pairs $(R, S)$ of tableaux of respective shapes $\rho$ and $\sigma$ with $R * S$ Knuthequivalent to $T$. See [Ful97, Ch. 5, Cor. 2(v)]. Similarly, $c_{\pi, \rho, \sigma}^{\tau}$ is the number of triples $(P, R, S)$ of tableaux of respective shapes $\pi, \rho$, and $\sigma$ with $P * R * S$ Knuth-equivalent to $T$.

Write $\operatorname{sh}(S)$ for the shape of a tableau $S$ and $S \equiv_{K} T$ if $S$ is Knuth-equivalent to $T$.

Lemma 5 Let $\sigma, \tau$ be partitions and fix a tableau $T$ of shape $\tau$. Then

$$
\Delta\left(s_{\tau / \sigma}\right)=\sum s_{\mathrm{sh}\left(R^{-}\right)} \otimes s_{\mathrm{sh}\left(R^{+}\right)},
$$

the sum taken over triples $\left(R^{-}, R^{+}, S\right)$ of tableaux with $\operatorname{sh}(S)=\sigma$ and $R^{-} * R^{+} * S \equiv_{K} T$. 
Note that $(\mu / \lambda)^{\prime}=\mu^{\prime} / \lambda^{\prime}$ and the operation $*$ makes sense for skew tableaux. If $S$ is a tableau of skew shape $\mu / \lambda$, put $|S|=|\mu / \lambda|=|\mu|-|\lambda|$.

Theorem 6 (Skew Littlewood-Richardson rule) Let $\lambda, \mu, \sigma, \tau$ be partitions and fix a tableau $T$ of shape $\tau$. Then

$$
s_{\mu / \lambda} \cdot s_{\tau / \sigma}=\sum(-1)^{\left|S^{-}\right|} s_{\mu^{+} / \lambda^{-}},
$$

the sum taken over triples $\left(S^{-}, S^{+}, S\right)$ of skew tableaux of respective shapes $\left(\lambda / \lambda^{-}\right)^{\prime}, \mu^{+} / \mu$, and $\sigma$ such that $S^{-} * S^{+} * S \equiv_{K} T$.

Remark 7 If $T$ is the unique Yamanouchi tableau of shape $\tau$ whose $i$ th row contains only the letter $i$, then this is almost Conjecture 6.1 in [AM]. Indeed, in this case $S$ is Yamanouchi of shape $\sigma$, so the sum is really over pairs of tableaux, and this explains the $\sigma$-Yamanouchi condition in [AM]. The difference lies in the tableau $S^{-}$and the reading word condition in [AM]. It is an exercise in tableaux combinatorics that there is a bijection between the indices $\left(S^{-}, S^{+}\right)$of Theorem 6 and the corresponding indices of Conjecture 6.1 in $[\mathrm{AM}$.

Proof Proof of Theorem 6; We reinterpret (15) in terms of tableaux. Let $\left(R^{-}, R^{+}, S\right)$ be a triple of tableaux of partition shape with $\operatorname{sh}(S)=\sigma$ and $R^{-} * R^{+} * S \equiv_{K} T$. If $\operatorname{sh}\left(R^{-}\right)=\rho$, then by [Ful97], Ch. 5, Cor. 2(i)], $c_{\lambda^{-}, \rho^{\prime}}^{\lambda}=c_{\left(\lambda^{-}\right)^{\prime}, \rho}^{\lambda^{\prime}}$ counts skew tableaux $S^{-}$of shape $\left(\lambda / \lambda^{-}\right)^{\prime}$ with $S^{-} \equiv_{K} R^{-}$. Likewise, if $\operatorname{sh}\left(R^{+}\right)=\pi$, then $c_{\mu, \pi}^{\mu^{+}}$counts skew tableaux $S^{+}$of shape $\mu^{+} / \mu$ with $S^{+} \equiv_{K} R^{+}$. Now 15] may be written as

$$
s_{\mu / \lambda} \cdot s_{\tau / \sigma}=\sum(-1)^{\left|S^{-}\right|} s_{\mu^{+} / \lambda^{-}},
$$

summing over skew tableaux $\left(R^{-}, R^{+}, S^{-}, S^{+}, S\right)$ with $R^{ \pm}$of partition shape, $\operatorname{sh}(S)=\sigma, R^{-} * R^{+} * S \equiv_{K}$ $T, \operatorname{sh}\left(S^{+}\right)=\mu^{+} / \mu, \operatorname{sh}\left(S^{-}\right)=\left(\lambda / \lambda^{-}\right)^{\prime}$, and $S^{ \pm} \equiv_{K} R^{ \pm}$.

Finally, note that $R^{ \pm}$is the unique tableau of partition shape Knuth-equivalent to $S^{ \pm}$. Since $S^{-} * S^{+} * S$ is Knuth-equivalent to $T$ (by transitivity of $\equiv_{K}$ ), we omit the unnecessary tableaux $R^{ \pm}$from the indices of summation and reach the statement of the theorem.

\section{Skew Littlewood-Richardson rule for Schur $P$ - and $Q$-functions}

The self-dual Hopf algebra of symmetric functions has a natural self-dual subalgebra $\Omega$. This has dual bases the Schur $P$ - and $Q$-functions [Mac95, III.8], which are indexed by strict partitions $\lambda: \lambda_{1}>\cdots>$ $\lambda_{l}>0$. Write $\ell(\lambda)=l$ for the length of the partition $\lambda$. As in Section 3 the constants and skew functions in the structure equations

$$
\begin{gathered}
Q_{\lambda} \cdot Q_{\mu}=\sum_{\nu} g_{\lambda, \mu}^{\nu} Q_{\nu} \Delta\left(Q_{\nu}\right)=\sum_{\lambda, \mu} f_{\lambda, \mu}^{\nu} Q_{\lambda} \otimes Q_{\mu}=\sum_{\mu} Q_{\nu / \mu} \otimes Q_{\mu} \\
\Delta\left(Q_{\tau / \sigma}\right)=\sum_{\pi, \rho} f_{\pi, \rho, \sigma}^{\tau} Q_{\pi} \otimes Q_{\rho}
\end{gathered}
$$

have combinatorial interpretations (see below). Also, each basis $\left\{P_{\lambda}\right\}$ and $\left\{Q_{\lambda}\right\}$ is almost self-dual in that $P_{\lambda}=2^{-\ell(\lambda)} Q_{\lambda}$ and $g_{\lambda, \mu}^{\nu}=2^{\ell(\lambda)+\ell(\mu)-\ell(\nu)} f_{\lambda, \mu}^{\nu}$. 
The algebra $\Omega$ is generated by the special $Q$-functions $q_{n}=Q_{(n)}:=\sum_{i+j=n} h_{i} e_{j}$ [Mac95, III, (8.1)]. This implies that $S\left(q_{n}\right)=(-1)^{n} q_{n}$, from which we deduce that

$$
S\left(Q_{\rho}\right)=(-1)^{|\rho|} Q_{\rho} .
$$

As $f_{\lambda^{-}, \rho}^{\lambda}=0$ unless $|\rho|=\left|\lambda / \lambda^{-}\right|$, we may write the algebraic rule (11) as

$$
Q_{\mu / \lambda} \cdot Q_{\tau / \sigma}=\sum_{\pi, \rho, \lambda^{-}, \mu^{+}}(-1)^{\left|\lambda / \lambda^{-}\right|} f_{\pi, \rho, \sigma}^{\tau} g_{\lambda^{-}, \rho}^{\lambda} g_{\mu, \pi}^{\mu^{+}} Q_{\mu^{+} / \lambda^{-}},
$$

with a similar identity holding for $P_{\mu / \lambda} \cdot P_{\tau / \sigma}$ (swapping $P \leftrightarrow Q$ and $f \leftrightarrow g$ ).

We formulate two combinatorial versions of (19). Strict partitions $\lambda, \mu$ are written as shifted Young diagrams (where row $i$ begins in column $i$ ). Skew shifted shapes $\lambda / \mu$ are defined in the obvious manner:

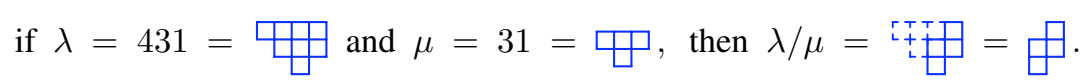

In what follows, tableaux means semi-standard (skew) shifted tableaux on a marked alphabet [Mac95, III.8]. We use shifted versions of the jeu-de-taquin and plactic equivalence from [Sag87] and [Ser], denoting the corresponding relations by $\equiv_{\mathrm{SJ}}$ and $\equiv_{\mathrm{SP}}$, respectively. Given tableaux $R, S, T$, we write $R * S \equiv_{\mathrm{SP}} T$ when representative words $u, v, w$ (built via "mread" [Ser, §2]) of the corresponding shifted plactic classes satisfy $u v \equiv_{\mathrm{SP}} w$.

Stembridge notes (following [Ste89, Prop. 8.2]) that for a fixed tableau $M$ of shape $\mu$,

$$
f_{\lambda, \mu}^{\nu}=\#\left\{\text { skew tableaux } L: \operatorname{sh}(L)=\nu / \lambda \text { and } L \equiv_{\text {SJ }} M\right\} .
$$

Serrano has a similar description of these coefficients in terms of $\equiv_{\mathrm{SP}}$. Fixing a tableau $T$ of shape $\tau$, it follows from [Ser, Cor. 1.15] that the coefficient $f_{\pi, \rho, \sigma}^{\tau}$ in $P_{\pi} \cdot P_{\rho} \cdot P_{\sigma}=\sum_{\tau} f_{\pi, \rho, \sigma}^{\tau} P_{\tau}$ is given by

$$
f_{\pi, \rho, \sigma}^{\tau}=\#\left\{(P, R, S): \operatorname{sh}(P)=\pi, \operatorname{sh}(R)=\rho, \operatorname{sh}(S)=\sigma, \text { and } P * R * S \equiv_{\mathrm{SP}} T\right\} .
$$

If $T$ is a tableau of shape $\lambda$, write $\ell(T)$ for $\ell(\lambda)$. The formula relating the $g$ 's and $f$ 's combines with 20) and 21) to give our next result.

Theorem 8 (Skew Littlewood-Richardson rule) Let $\lambda, \mu, \sigma, \tau$ be strict partitions and fix a tableau $T$ of shape $\tau$. Then

$$
Q_{\mu / \lambda} \cdot Q_{\tau / \sigma}=\sum(-1)^{\left|\lambda / \lambda^{-}\right|} 2^{\ell\left(R^{-}\right)+\ell\left(R^{+}\right)+\ell\left(\lambda^{-}\right)+\ell(\mu)-\ell(\lambda)-\ell\left(\mu^{+}\right)} Q_{\mu^{+} / \lambda^{-}},
$$

the sum taken over quintuples $\left(R^{-}, R^{+}, S^{-}, S^{+}, S\right)$ with $R^{ \pm}$of partition shape, $\operatorname{sh}(S)=\sigma, R^{-} * R^{+} *$ $S \equiv_{\mathrm{SP}} T, \operatorname{sh}\left(S^{+}\right)=\mu^{+} / \mu, \operatorname{sh}\left(S^{-}\right)=\left(\lambda / \lambda^{-}\right)$, and $S^{ \pm} \equiv_{\mathrm{SJ}} R^{ \pm}$.

Serrano conjectures an elegant combinatorial description [Ser, Conj. 2.12 and Cor. 2.13] of the structure constants $g_{\lambda, \mu}^{\nu}$ in (17): For any tableau $M$ of shape $\mu$, he conjectured

$$
g_{\lambda, \mu}^{\nu}=\#\left\{\text { skew tableaux } L: \operatorname{sh}(L)=\nu / \lambda \text { and } L \equiv_{\mathrm{SP}} M\right\} .
$$

(Note that if $S, T$ are tableaux, then $S \equiv_{\mathrm{SP}} T$ does not necessarily imply that $S \equiv_{\mathrm{SJ}} T$.) This leads to a conjectural reformulation of Theorem 8 in the spirit of Theorem 6 . 
Conjecture 1 (Conjectural Skew Littlewood-Richardson rule) Let $\lambda, \mu, \sigma, \tau$ be strict partitions, and fix a tableau $T$ of shape $\tau$. Then

$$
Q_{\mu / \lambda} \cdot Q_{\tau / \sigma}=\sum(-1)^{\left|S^{-}\right|} Q_{\mu^{+} / \lambda^{-}},
$$

the sum taken over triples $\left(S^{-}, S^{+}, S\right)$ of skew tableaux of respective shapes $\left(\lambda / \lambda^{-}\right), \mu^{+} / \mu$, and $\sigma$ such that $S^{-} * S^{+} * S \equiv_{\mathrm{SP}} T$.

Proof: There is a unique shifted tableau $R$ in any shifted plactic class [Ser, Thm. 2.8]. So the conditions $S^{ \pm} \equiv_{\mathrm{SP}} R^{ \pm}$and $R^{-} * R^{+} * S \equiv_{\mathrm{SP}} T$ in 21) and 23) may be replaced with the single condition $S^{-} * S^{+} * S \equiv_{\mathrm{SP}} T$.

\section{Skew Littlewood-Richardson rule for noncommutative Schur functions}

The Hopf algebra of noncommutative symmetric functions was introduced, independently, in $\mathrm{GKL}^{+} 95$, MR95] as the (graded) dual to the commutative Hopf algebra of quasisymmetric functions. We consider the dual bases (indexed by compositions) $\left\{F_{\alpha}\right\}$ of Gessel's quasisymmetric functions and $\left\{R_{\alpha}\right\}$ of noncommutative ribbon Schur functions. The structure constants in

$$
\begin{gathered}
R_{\alpha} \cdot R_{\beta}=\sum_{\gamma} b_{\alpha, \beta}^{\gamma} R_{\gamma} \quad \Delta\left(R_{\gamma}\right)=\sum_{\alpha, \beta} c_{\alpha, \beta}^{\gamma} R_{\alpha} \otimes R_{\beta}=\sum_{\beta} R_{\gamma / \beta} \otimes R_{\beta} \\
\Delta\left(R_{\tau / \sigma}\right)=\sum_{\pi, \rho, \sigma} c_{\pi, \rho, \sigma}^{\tau} R_{\pi} \otimes R_{\rho}
\end{gathered}
$$

may be given combinatorial meaning via the descent map $\mathrm{d}: \mathfrak{S}_{n} \rightarrow \Gamma_{n}$ from permutations to compositions and a section of it w: $\Gamma_{n} \rightarrow \mathfrak{S}_{n}$. These maps are linked via ribbon diagrams, edge-connected skew Young diagrams (written in the french style), with no $2 \times 2$ subdiagram present. By way of example,

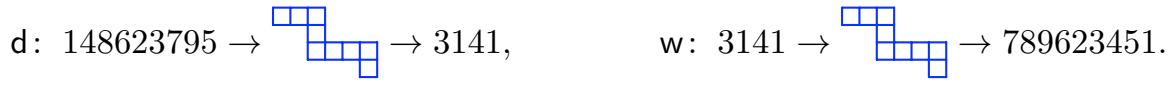

(In the intermediate step for $\mathrm{d}(w)$, new rows in the ribbon begin at descents of $w$. In the intermediate step for $\mathrm{w}(\alpha)$, the boxes in the ribbon are filled left-to-right, bottom-to-top.)

A ribbon $\alpha$ may be extended by a ribbon $\beta$ in two ways: affixing $\beta$ to the rightmost edge or bottommost edge of $\alpha$ (written $\alpha \triangleleft \beta$ and $\alpha \Delta \beta$, respectively):

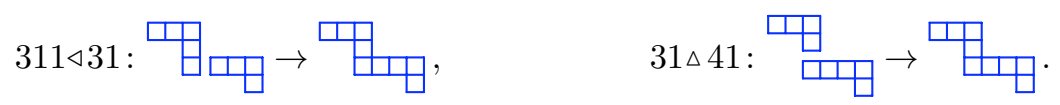

If a ribbon $\gamma$ is formed from $\alpha$ and $\beta$ in either of these two ways, we write $\gamma \in \alpha \diamond \beta$. The coefficient $b_{\alpha, \beta}^{\gamma}$ is 1 , if $\gamma \in \alpha \diamond \beta$, and 0 otherwise. If $*$ is the shifted shuffle product on permutations (see (3.4) in [MR95]), then the coefficient $c_{\alpha, \beta}^{\gamma}$ is the number of words $w$ in $\mathrm{w}(\alpha) * \mathrm{w}(\beta)$ such that $\mathrm{d}(w)=\gamma$. The coefficient $c_{\pi, \rho, \sigma}^{\tau}$ has the analogous description. 
Antipode formulas for the distinquished bases were found, independently, in [Ehr96, MR95]:

$$
S\left(F_{\alpha}\right)=(-1)^{|\alpha|} F_{\alpha^{\prime}} \quad \text { and } \quad S\left(R_{\alpha}\right)=(-1)^{|\alpha|} R_{\alpha^{\prime}},
$$

where $\alpha^{\prime}$ is the conjugate of $\alpha$ (in the sense of french style skew partitions). For example, $(3141)^{\prime}=$ 211311. The descriptions of the antipode and structure constants in 25) and 26) give a formula for the product of two skew ribbon Schur functions.

Theorem 9 (Skew Littlewood-Richardson rule) Let $\alpha, \beta, \sigma, \tau$ be compositions. Then

$$
R_{\beta / \alpha} \cdot R_{\tau / \sigma}=\sum(-1)^{|\rho|} R_{\beta^{+} / \alpha^{-}},
$$

the sum taken over factorizations $\alpha \in \alpha^{-} \diamond \rho^{\prime}$, extensions $\beta^{+} \in \beta \diamond \pi$, and words $w$ in the shuffle product $\mathrm{w}(\pi) * \mathrm{w}(\rho) * \mathrm{w}(\sigma)$ such that $\mathrm{d}(w)=\tau$.

Remark 10 The nonzero skew ribbon Schur functions $R_{\beta / \alpha}$ do not correspond to skew ribbon shapes in a simple way. For example, 111 is not a (connected) sub-ribbon of 221 , yet $R_{221 / 111}=R_{2}+R_{11} \neq 0$. Contrast this with the skew functions $F_{\beta / \alpha}$, where $F_{\beta / \alpha} \neq 0$ if and only if $\beta \in \omega \diamond \alpha$ for some ribbon $\omega$. That is, $F_{\beta / \alpha}=F_{\omega}$. Thus we may view $\alpha$ as the last $|\alpha|$ boxes of the ribbon $\beta$ and $\beta / \alpha$ as the complementary ribbon $\omega$. Interpreting $F_{\beta / \alpha} \cdot F_{\tau / \sigma}$ alternately as a product of ordinary functions or skew functions yields the curious identity

$$
F_{\beta / \alpha} \cdot F_{\tau / \sigma}=\sum_{\gamma} c_{\beta / \alpha, \tau / \sigma}^{\gamma} F_{\gamma}=\sum_{\pi, \rho, \alpha^{-}, \beta^{+}}(-1)^{|\rho|} b_{\pi, \rho}^{\tau / \sigma} c_{\alpha^{-}, \rho^{\prime}}^{\alpha} c_{\beta, \pi}^{\beta^{+}} F_{\beta^{+} / \alpha^{-}} .
$$

\section{Skew $k$-Pieri rule for $k$-Schur functions}

Fix an integer $k \geq 1$. Let $\Lambda_{(k)}$ denote the Hopf subalgebra of the Hopf algebra of symmetric functions generated by the homogeneous symmetric functions $h_{1}, h_{2}, \ldots, h_{k}$. Let $\Lambda^{(k)}$ denote the Hopf-dual quotient Hopf algebra of symmetric functions. We consider the dual bases $\left\{s_{\lambda}^{(k)}\right\} \subset \Lambda_{(k)}$ and $\left\{F_{\lambda}^{(k)}\right\} \subset \Lambda^{(k)}$ of $k$-Schur functions and dual $k$-Schur functions of [LLMS, LM07], also called strong Schur functions and weak Schur functions in [LLMS]. The $k$-Schur functions were first introduced by Lapointe, Lascoux, and Morse in the context of Macdonald polynomials, and were later shown by Lam to represent Schubert classes in the affine Grassmannian of $\operatorname{SL}(k+1, \mathbb{C})$. We refer the reader to the references in [LLMS].

Here $\lambda$ varies over all $k$-bounded partitions, that is, those partitions satisfying $\lambda_{1} \leq k$. There is an involution $\lambda \mapsto \lambda^{\omega_{k}}$ on $k$-bounded partitions called $k$-conjugation. We have

$$
S\left(s_{\lambda}^{(k)}\right)=(-1)^{|\lambda|} s_{\lambda^{\omega_{k}}}^{(k)} \quad \text { and } \quad S\left(F_{\lambda}^{(k)}\right)=(-1)^{|\lambda|} F_{\lambda \omega_{k}}^{(k)} .
$$

If $\lambda=(r)$ is a one-part partition, then $s_{\lambda}^{(k)}=h_{r}$ is a homogeneous symmetric function. We have the $k$-Pieri and dual $k$-Pieri rules [LLMS, LM07] (called weak and strong Pieri rules in [LLMS])

$$
s_{\lambda}^{(k)} \cdot h_{r}=\sum_{\lambda \rightsquigarrow_{r} \mu} s_{\mu}^{(k)} \quad \text { and } \quad F_{\lambda}^{(k)} \cdot h_{r}=\sum_{\lambda \rightarrow_{r} \mu} F_{\mu}^{(k)}
$$

for $r \leq k$. Here $\lambda \rightsquigarrow_{r} \mu$ denotes an $r$-weak strip connecting $\lambda$ and $\mu$-present if and only if both $\mu / \lambda$ and $\mu^{\omega_{k}} / \lambda^{\omega_{k}}$ are horizontal $r$-strips. The notation $\lambda \rightarrow_{r} \mu$ denotes an $r$-strong strip as introduced in [LLMS], 
which we will not define here. The terminology comes from the relationship with the weak and strong (Bruhat) orders of the affine symmetric group. We remark that there may be distinct strong strips $\lambda \rightarrow_{r} \mu$ and $\left(\lambda \rightarrow_{r} \mu\right)^{\prime}$ which start and end at the same partition, so that the second Pieri rule of (28) may have multiplicities. (Strictly speaking, the strong strips in [LLMS] are built on $(k+1)$-cores, and our $\lambda \rightarrow_{r} \mu$ denotes the strips obtained after applying a bijection between $(k+1)$-cores and $k$-bounded partitions.)

We define skew functions $s_{\lambda / \mu}^{(k)}$ and $F_{\lambda / \mu}^{(k)}$ using (7) and (8). There is an explicit combinatorial description of $F_{\lambda / \mu}^{(k)}$ in terms of the weak tableaux of [LLMS], but only a conjectured combinatorial description of $s_{\lambda / \mu}^{(k)}$ [LLMS, Conj. 4.18(3)].

Theorem 11 (Skew $k$-Pieri (or weak Pieri) rule) For $k$-bounded partitions $\lambda, \mu$, and $r \leq k$,

$$
s_{\mu / \lambda}^{(k)} \cdot h_{r}=\sum_{i+j=r}(-1)^{j} \sum_{\substack{\mu \rightsquigarrow i \\\left(\lambda^{-}\right)^{\omega_{k}} \mu_{j}^{+} \lambda^{\omega_{k}}}} s_{\mu^{+} / \lambda^{-}}^{(k)}
$$

Proof: In Theorem 3 , take $L_{\tau / \sigma}=h_{r}$. For $c_{\pi, \rho, \sigma}^{\tau}$, use the formula $\Delta\left(h_{r}\right)=\sum_{i+j=r} h_{i} \otimes h_{j}$, and for $b_{\lambda^{-}, \rho^{\omega_{k}}}^{\lambda}$ and $b_{\mu, \pi}^{\mu^{+}}$, use 28 .

Theorem 12 (Skew dual $k$-Pieri (or strong Pieri) rule) For $k$-bounded partitions $\lambda$, $\mu$, and $r \leq k$,

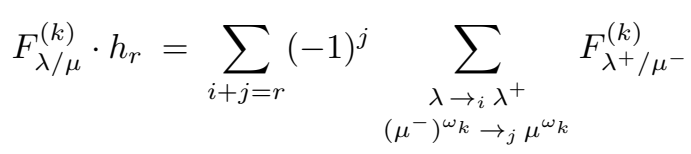

Proof: Identical to the proof of Theorem 11 .

As an example, let $k=2, r=2, \mu=(2,1,1)$, and $\lambda=(1)$. Then Theorem 11 states that

$$
s_{211 / 1}^{(2)} \cdot h_{2}=s_{2211 / 1}^{(2)}-s_{2111}^{(2)},
$$

which one can verify using [28) and the expansions $s_{211 / 1}^{(2)}=s_{21}^{(2)}+s_{111}^{(2)}$ and $s_{2211 / 1}^{(2)}=2 s_{2111}^{(2)}+s_{221}^{(2)}$. Theorem 12 states that

$$
F_{211 / 1}^{(2)} \cdot h_{2}=3 F_{222 / 1}^{(2)}+5 F_{2211 / 1}^{(2)}+3 F_{21111 / 1}^{(2)}+3 F_{111111 / 1}^{(2)}-2 F_{221}^{(2)}-3 F_{2111}^{(2)}-2 F_{11111}^{(2)} .
$$

One can verify that both sides are equal to $6 F_{221}^{(2)}+5 F_{2111}^{(2)}+4 F_{11111}^{(2)}$.

\section{Skew Pieri rule for affine Grassmannian of the symplectic group}

Fix $n \geq 1$. The Hopf algebra $\Omega$ of Section 4 contains a Hopf subalgebra $\Omega_{(n)}$ generated by the Schur $P$-functions $P_{1}, P_{3}, \ldots, P_{2 n-1}$. In [LSS10], it was shown that $\Omega_{(n)}$ is isomorphic to the homology ring $H_{*}\left(G r_{\mathrm{Sp}(2 n, \mathbb{C})}\right)$ of the affine Grassmannian of the symplectic group $\operatorname{Sp}(2 n, \mathbb{C})$. A distinguished basis 
$\left\{P_{w}^{(n)}\right\} \subset \Omega_{(n)}$, representing the Schubert basis, was studied there. The symmetric functions $P_{w}^{(n)}$ are shifted versions of the $k$-Schur functions of Section 6 .

The indexing set for the basis $\left\{P_{w}^{(n)}\right\}$ is the set $\widetilde{C}_{n}^{0}$ of affine Grassmannian type $C$ permutations: they are the minimal length coset representatives of $C_{n}$ in $\widetilde{C}_{n}$. A lower Bruhat order ideal $\mathcal{Z} \subset \widetilde{C}_{n}$ of the affine type $C$ Weyl group is defined in [LSS10]. Let $\mathcal{Z}_{j} \subset \mathcal{Z}$ denote those $v \in \mathcal{Z}$ with length $\ell(v)=j$. For each $v \in \mathcal{Z}$, there is a nonnegative integer $c(v) \in \mathbb{Z}_{\geq 0}$, called the number of components of $v$. We note that $c(\mathrm{id})=0$. With this notation, for each $1 \leq j \leq 2 n-1$, we have the Pieri rule [LSS10, Thms. 1.3 and 1.4]

$$
P_{w}^{(n)} \cdot P_{j}=\sum_{v \in \mathcal{Z}_{j}} 2^{c(v)-1} P_{v w}^{(n)},
$$

where the sum is over all $v \in \mathcal{Z}_{j}$ such that $v w \in \widetilde{C}_{n}^{0}$, and $\ell(v w)=\ell(v)+\ell(w)$.

It follows from the discussion in Section 4 that the antipode acts on the $P_{j}$ by $S\left(P_{j}\right)=(-1)^{j} P_{j}$. We define $P_{w / v}^{(n)}$ using 77 .

Theorem 13 (Skew Pieri rule) For $w, v \in \widetilde{C}_{n}^{0}$, and $r \leq 2 n-1$,

$$
P_{w / v}^{(n)} \cdot P_{r}=\sum_{i+j=r}(-1)^{j} \sum_{\substack{u \in \mathcal{Z}_{i} \\ z \in \mathcal{Z}_{j}}} 2^{c(u)+c(z)-1} P_{u w / z^{-1} v}^{(n)},
$$

where the sum is over all $u \in \mathcal{Z}_{i}$ and $z \in \mathcal{Z}_{j}$ such that $u w, z^{-1} v \in \widetilde{C}_{n}^{0}, \ell(u w)=\ell(u)+\ell(w)$, and $\ell\left(z^{-1} v\right)+\ell(z)=\ell(v)$.

Proof: In Theorem 3, take $L_{\tau / \sigma}=P_{r}$ and use 29]. For the constants $c_{\pi, \rho, \sigma}^{\tau}$, use the formula

$$
\Delta\left(P_{r}\right)=1 \otimes P_{r}+P_{r} \otimes 1+2 \sum_{0<j<r} P_{r-j} \otimes P_{j} .
$$

If $0<j<r$, the product $c_{\pi, \rho, \sigma}^{\tau} b_{\lambda^{-}, \rho^{\prime}}^{\lambda} b_{\mu, \pi}^{\mu^{+}}$in 11 becomes $2 \cdot 2^{c(u)-1} \cdot 2^{c(z)-1}=2^{c(u)+c(z)-1}$. If $j=0$ (resp., $j=r$ ), it becomes $1 \cdot 2^{c(u)-1} \cdot 1=2^{c(u)+c(z)-1}$ (resp., $1 \cdot 1 \cdot 2^{c(z)-1}=2^{c(u)+c(z)-1}$ ).

\section{References}

[AM] Sami H. Assaf and Peter R. McNamara. A Pieri rule for skew shapes. J. Combin. Theory Ser. A. To appear.

[Ehr96] Richard Ehrenborg. On posets and Hopf algebras. Adv. Math., 119(1):1-25, 1996.

[Fu197] William Fulton. Young tableaux, volume 35 of London Mathematical Society Student Texts. Cambridge University Press, Cambridge, 1997.

[GKL $\left.{ }^{+} 95\right]$ Israel M. Gelfand, Daniel Krob, Alain Lascoux, Bernard Leclerc, Vladimir S. Retakh, and Jean-Yves Thibon. Noncommutative symmetric functions. Adv. Math., 112(2):218-348, 1995. 
[LLMS] Thomas Lam, Luc Lapointe, Jennifer Morse, and Mark Shimozono. Affine insertion and Pieri rules for the affine Grassmannian. Memoirs of the AMS. To appear.

[LM07] Luc Lapointe and Jennifer Morse. A $k$-tableau characterization of $k$-Schur functions. Adv. Math., 213(1):183-204, 2007.

[LSS10] Thomas Lam, Anne Schilling, and Mark Shimozono. Schubert polynomials for the affine Grassmannian of the symplectic group. Math. Z., 264(4):765-811, 2010.

[Mac95] I. G. Macdonald. Symmetric functions and Hall polynomials. Oxford Mathematical Monographs. The Clarendon Press Oxford University Press, New York, second edition, 1995.

[Mon93] Susan Montgomery. Hopf algebras and their actions on rings, volume 82 of CBMS Regional Conference Series in Mathematics. Published for the Conference Board of the Mathematical Sciences, Washington, DC, 1993.

[MR95] Claudia Malvenuto and Christophe Reutenauer. Duality between quasi-symmetric functions and the Solomon descent algebra. J. Algebra, 177(3):967-982, 1995.

[Sag87] Bruce E. Sagan. Shifted tableaux, Schur $Q$-functions, and a conjecture of R. Stanley. $J$. Combin. Theory Ser. A, 45(1):62-103, 1987.

[Ser] Luis Serrano. The shifted plactic monoid. Math. Z. To appear.

[Ste89] John R. Stembridge. Shifted tableaux and the projective representations of symmetric groups. Adv. Math., 74(1):87-134, 1989.

[Zel81] Andrey V. Zelevinsky. Representations of finite classical groups. A Hopf algebra approach, volume 869 of Lecture Notes in Mathematics. Springer-Verlag, Berlin, 1981. 
